\title{
Influence of serum testosterone on urinary continence and sexual activity in patients undergoing radical prostatectomy for clinically localized prostate cancer
}

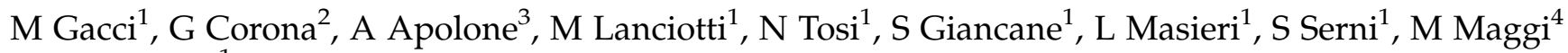 \\ and M Carini ${ }^{1}$ \\ ${ }^{1}$ Department of Urology, University of Florence, Careggi Hospital, Florence, Italy; ${ }^{2}$ Endocrinology Unit, Maggiore-Bellaria Hospital, \\ Bologna, Italy; ${ }^{3}$ Department of Oncology, Center for the Evaluation and Research on Pain (CERP), Istituto di Ricerche \\ Farmacologiche Mario Negri, Milan, Italy and ${ }^{4}$ Andrology Unit, Department of Clinical Physiopathology, University of Florence, \\ Florence, Italy
}

\begin{abstract}
The aim of the present study was to evaluate how serum testosterone level $(T)$ can affect urinary continence and erectile function in patients undergoing radical prostatectomy (RP). We included 257 patients with clinically localized prostate cancer, those who had filled out preoperative quality of life questionnaires (University of California, Los Angeles Prostate Cancer Index, International Index of Erectile Function (IIEF)), and those who had $T$ and total PSA sampled the day before surgery. We calculated correlations between $T$ and age, body mass index (BMI), PSA, urinary function or bother (UF, UB) and sexual function or bother (SF, SB) and IIEF-5 in the whole population and in sub-populations with normal $\left(\geqslant 10.4 \mathrm{nmoll}^{-1}\right)$ and low $\left(<10.4 \mathrm{ng} \mathrm{ml}^{-1}\right) \mathrm{T}$ using Pearson's and Spearman's correlation coefficients. We evaluated differences in these parameters between patients with low and normal Tusing the unpaired samples $t$-test and Mann-Whitney test, and finally the correlation between UF and SF, UB and SB, and between PSA and $T$ in the overall population, and separately in patients with low and normal $T$ using the Pearson's correlation coefficient. Mean preoperative $T$ was $13.5 \mathrm{nmol}^{-1}$ and $23.7 \%$ of patients presented a low $T$. Mean age, mean BMI and mean preoperative total PSA at RP were 64.3 years, $25.9 \mathrm{~kg} \mathrm{~m}^{-2}$ and $9.0 \mathrm{ng} \mathrm{ml}^{-1}$, respectively. BMI was negatively correlated with $T$ in the overall population $(r=-0.266 ; P=0.02)$; moreover, patients with normal $T$ presented lower BMI compared with patients with low $T$ (25.7 vs 27.6: $P=0.02$ ). We found a significant correlation between $S F$ scores and $T$ in patients with normal $T$ $(r=0.1777: P=0.05)$. SF was significantly higher in patients with normal $T$ compared with those with low $T$ (74.8 vs $64.8: P=0.05)$. Furthermore, UF and UB were significantly correlated with $S F$ $(r=0.2544: P<0.01)$ and SB $(r=0.2512: P=0.01)$, respectively, in men with normal $T$. Serum $T$ was significantly correlated with PSA in men with low $T(r=0.3874: P=0.0029)$, whereas this correlation was missed in the whole population and in men with normal $T$. The correlation between preoperative PSA and $T$ in men with low $T$ is in agreement with the 'saturation' model proposed by Morgentaler. The correlation between basal $T$ and preoperative erectile function and urinary continence underlines the importance of assessing $T$ before RP.

Prostate Cancer and Prostatic Diseases (2010) 13, 168-172; doi:10.1038/pcan.2010.4; published online 9 March 2010
\end{abstract}

Keywords: testosterone; free testosterone; hypogonadism

\section{Introduction}

The quality of life (QOL) of patients with prostate cancer (PCa) is usually assessed to measure the safety and tolerability of treatment outcomes, ${ }^{1,2}$ to compare different therapeutic approaches ${ }^{3}$ or to monitor the progression of disease during time. ${ }^{4}$

Correspondence: Dr M Gacci, Urology I, University of Florence, Via Masaccio 102, Florence 50139, Italy.

E-mail: maurogacci@yahoo.it

Received 2 December 2009; revised 29 January 2010; accepted 29 January 2010; published online 9 March 2010
Moreover, several authors have reported that the return of serum testosterone level $(T)$ to normal range during intermittent androgen deprivation is associated with a significant improvement of general well-being, and urinary (UF) and sexual function (SF) ${ }^{5-7}$ In particular, QOL benefits in the off-treatment phase are shown by a shift toward baseline levels in the scales depicting physical and work functions, urgency, nocturia, impotence and sexual performance. ${ }^{8}$

Furthermore, hypogonadism is very common in elderly men and men who have PCa: the symptoms of hypogonadism, such as depression, erectile dysfunction (ED) and lower urinary tract symptoms, can significantly 
impair a man's QOL. ${ }^{9}$ In a recent retrospective study, such as in several recent reviews, the authors suggested that testosterone replacement therapy can be achieved in hypogonadal men undergoing treatments for PCa. ${ }^{10-13}$

The aim of the present study is to evaluate the influence of serum testosterone on urinary continence and sexual activity in patients undergoing radical prostatectomy (RP) for clinically localized PCa.

\section{Materials and methods}

\section{Population}

A total of 257 patients were consecutively selected for the study. Inclusion criteria were as follows: having undergone RP as primary therapy for clinically localized PCa (cT1-cT2, N0, M0); having preoperatively filled out the QOL questionnaires (University of California, Los Angeles Prostate Cancer Index (UCLA-PCI) and international index of erectile function (IIEF)); having sampled the testosterone and total PSA levels the day before surgery; and having obtained signed informed consent. Patients treated with neoadjuvant hormone or radiotherapy, with missing data, with an inability to complete the questionnaire and refusal to participate, without preoperative testosterone or PSA, were excluded from the trial.

Age, body mass index (BMI; weight/height ${ }^{2}$ ), testosterone and PSA were preoperatively recorded at about 0800 hours in a recovery regiment the day before the RP.

\section{HRQOL measures}

Informed consent was obtained from all subjects. This trial was carried out in accordance with the ethical principles of the Helsinki Declaration (1996) and Good Clinical Practice Issues (1997) and was reviewed and approved by both the local ethics committees.

We used the validated Italian version of the UCLA-PCI and the IIEF-5 to assess urinary and sexual QOL condition. UCLA-PCI allows evaluation of the detailed symptoms as well as their corresponding bother. For this analysis, we focused on subject UF and SF and urinary and sexual bother (UB and SB). Responses were scored from 0 to 100 , with a higher score indicating better QOL. ${ }^{14}$

IIEF was used to better define the erectile function. The possible scores for the IIEF-5 range from 5 to 25, and ED was classified into five categories based on the scores: severe (5-7), moderate (8-11), mild to moderate (12-16), mild (17-21) and no ED (22-25). ${ }^{15}$

\section{Statistics}

We calculated the correlations between $T$ and age, BMI, PSA, UF, UB, SF, SB and IIEF-5 in the whole population and in both the subpopulations of men with normal $\left(T \geqslant 10.4 \mathrm{nmoll}^{-1}\right)$ and low $\left(T<10.4 \mathrm{ng} \mathrm{ml}^{-1}\right)$ serum $T$ using the Pearson's correlation coefficient and Spearman's correlation coefficient (see Table 1).

Moreover, we evaluated the differences in age, BMI, PSA, UF, UB, SF, SB and IIEF-5 between patients with $T<10.4 \mathrm{nmoll}^{-1}$ and $T \geqslant 10.4 \mathrm{nmoll}^{-1}$ using an unpaired samples $t$-test and a Mann-Whitney test (see Table 2).

Finally, we evaluated the correlation between UF and SF, UB and SB, and the correlation between PSA and testosterone in the overall population and separately in patients with low and normal $T$ using the Pearson's correlation coefficient (see Figures 1 and 2).

\section{Results}

Mean preoperative $T$ was $13.5 \mathrm{nmoll}^{-1}$ (median $13.2 \mathrm{nmoll}^{-1}$, range $\left.0.5-29\right)$ : 61 patients $(23.7 \%)$ had a $T<10.4$ nmoll $^{-1}$. Mean age at RP was 64.3 years (median 67 , range $45-76$ ). Mean BMI was $25.9 \mathrm{~kg} \mathrm{~m}^{-2}$ (median 25.5, range $17.7-34.6)$ : 10 subjects $(3.9 \%)$ had BMI $<20 \mathrm{~kg} \mathrm{~m}^{-2}, 90(35.0 \%)$ between 20 and $24.9 \mathrm{~kg} \mathrm{~m}^{-2}$ and $157(61.1 \%)$ above $25 \mathrm{~kg} \mathrm{~m}^{-2}$. Mean preoperative total PSA was $9.0 \mathrm{ng} \mathrm{ml}^{-1}$ (median 7.6, range 0.6-48.8): 187 $(72.7 \%)$ had a PSA $<10 \mathrm{ng} \mathrm{ml}^{-1}, 53(20.6 \%)$ between $10-$ $20 \mathrm{ng} \mathrm{ml}^{-1}$ and $17(6.6 \%)$ above $20 \mathrm{ng} \mathrm{ml}^{-1}$. Mean UCLAPCI and IIEF scores in the overall population and in patients with normal and low $T$ are reported in Table 2.

We did not report any significant results from the analyses of age. BMI was negatively correlated with $T$ in the overall population (see Table 1: $P=0.02$ ); moreover, patients with normal $T$ presented lower BMI compared with patients with $T$ below 10.4 nmol $^{-1}$ (see Table 2: 25.7 vs $\left.27.6 \mathrm{~kg} \mathrm{~m}^{-2}, P=0.02\right)$.

We evidenced a significant correlation between SF scores and $T$ in patients with $T$ above $10.4 \mathrm{nmoll}^{-1}$ (see Table 1: $P=0.05)$. Moreover, $\mathrm{SF}$ was significantly higher in patients with normal $T$ compared with patients with low $T$ (see Table 2: 74.8 vs $64.8 ; P=0.05$ ). The remaining UCLA-PCI items (UF, UB, SB) and the IIEF questionnaire were statistically insignificant in all the analyses.

Table 1 Correlations between testosterone and age, BMI, PSA, UF, UB, SF, SB (items from UCLA-PCI) and IIEF-5 in the whole population (overall) and in both the subpopulations of hypogonadal $(T<10.4)$ and eugonadal $(T \geqslant 10.4)$ men using Pearson's and Spearman's correlation coefficients

\begin{tabular}{|c|c|c|c|c|c|c|c|c|c|}
\hline & No. of pts (\%) & $A g e^{\mathrm{a}}$ & $B M I^{a}$ & $P S A^{\mathrm{a}}$ & $U F$ & $U B$ & $S F$ & $S B$ & $I I E F^{a}$ \\
\hline Overall & 257 & $\begin{aligned} r & =0.033 \\
P & =0.6024\end{aligned}$ & $\begin{array}{c}r=-0.266 \\
\boldsymbol{P}=\mathbf{0 . 0 2 1 3}\end{array}$ & $\begin{array}{c}r=-0.035 \\
P=0.5802\end{array}$ & $\begin{array}{l}r=0.0173 \\
P=0.8479\end{array}$ & $\begin{array}{c}r=-0.1000 \\
P=0.2670\end{array}$ & $\begin{array}{c}r=-0.0297 \\
P=0.7444\end{array}$ & $\begin{array}{l}r=0.0102 \\
P=0.9107\end{array}$ & $\begin{array}{l}r=-0.054 \\
P=0.4540\end{array}$ \\
\hline $\mathrm{T}<10.4$ & $61(23.7)$ & $\begin{aligned} r & =0.054 \\
P & =0.6850\end{aligned}$ & $\begin{array}{c}r=-0.121 \\
P=0.6623\end{array}$ & $\begin{aligned} r & =0.360 \\
\boldsymbol{P} & =\mathbf{0 . 0 0 6 6}\end{aligned}$ & $\begin{array}{c}r=-0.0318 \\
P=0.8912\end{array}$ & $\begin{array}{c}r=-0.1281, \\
P=0.5799\end{array}$ & $\begin{array}{l}r=0.1179 \\
P=0.6308\end{array}$ & $\begin{array}{l}r=0.3769 \\
P=0.1117\end{array}$ & $\begin{aligned} r & =0.124 \\
P & =0.4326\end{aligned}$ \\
\hline $\mathrm{T} \geqslant 10.4$ & $196(76.3)$ & $\begin{aligned} r & =0.043 \\
P & =0.5546\end{aligned}$ & $\begin{array}{c}r=-0.100 \\
P=0.4335\end{array}$ & $\begin{array}{l}r=-0.107 \\
P=0.1396\end{array}$ & $\begin{array}{l}r=0.0682 \\
P=0.4895\end{array}$ & $\begin{array}{c}r=-0.0552 \\
P=0.5779\end{array}$ & $\begin{array}{l}r=0.1777 \\
P=\mathbf{0 . 0 5 1 1}\end{array}$ & $\begin{array}{l}r=0.1360 \\
P=0.1686\end{array}$ & $\begin{aligned} r & =0.026 \\
P & =0.7507\end{aligned}$ \\
\hline
\end{tabular}

Abbreviations: BMI, body mass index; IIEF, International Index of Erectile Function; SB, sexual bother; SF, sexual function; UB, urinary bother; UCLA-PCI, University of California Los Angeles Prostate Cancer Index; UF, urinary function.

${ }^{a}$ Calculated using Spearman's correlation coefficient. Bold entries are data with statistical significance. 
Table 2 Differences in age, PSA, UF, UB, SF, SB and IIEF-5 between hypogonadal $(T<10.4)$ and eugonadal $(T \geqslant 10.4)$ men: mean value according to groups, and levels of significance $(T<10.4$ vs $T \geqslant 10.4)$ using the unpaired samples $t$-test and Mann-Whitney test

\begin{tabular}{lccccccccc}
\hline & No. of pts (\%) & $A g e^{\mathrm{a}}$ & $B M I^{\mathrm{a}}$ & $P S A^{\mathrm{a}}$ & $U F$ & $U B$ & $S F$ & $S B$ & IIEF \\
\hline Overall & 257 & 64.3 & 25.9 & 9.0 & 94.9 & 92.4 & 72.9 & 71.6 \\
$T<10.4$ & $61(23.7)$ & 64.1 & 27.6 & 8.9 & 96.1 & 95.2 & 64.8 & 61.8 \\
$T \geqslant 10.4$ & $196(76.3)$ & 64.3 & 25.7 & 9.0 & 94.5 & 91.6 & 74.8 & 73.6 \\
$P$ & & $P=0.8888$ & $\boldsymbol{P}=\mathbf{0 . 0 1 1 8}$ & $P=0.8920$ & $P=0.5905$ & $P=0.3423$ & $\boldsymbol{P}=\mathbf{0 . 0 5 4 3}$ & $P=0.1608$ & $P=0.4155$ \\
\hline
\end{tabular}

Abbreviations: BMI, body mass index; IIEF, International Index of Erectile Function; SB, sexual bother; SF, sexual function; UB, urinary bother; UF, urinary function.

${ }^{a}$ Calculated using Mann-Whitney test. Bold entries are data with statistical significance.

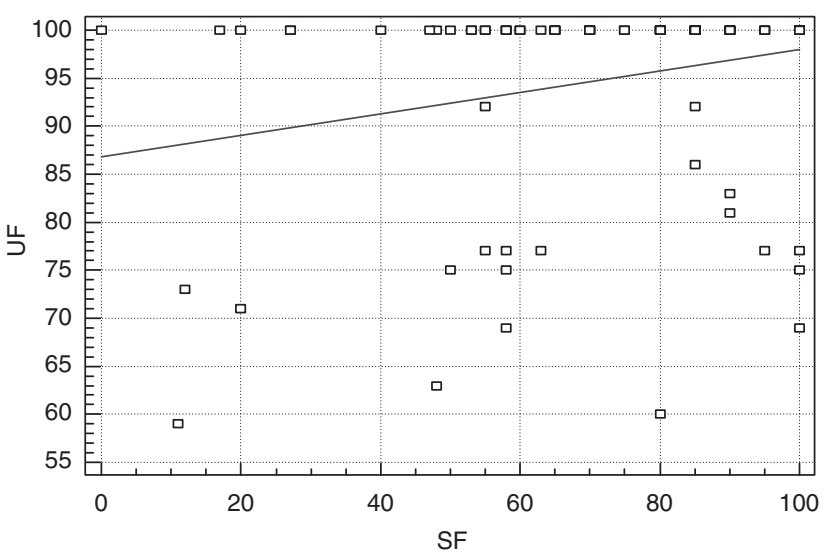

Figure 1 Correlation between urinary function (UF) and sexual function $(\mathrm{SF})$ in men with $T \geqslant 10.4 \mathrm{nmoll}^{-1}$. Pearson's correlation coefficient: $r=0.2544 ; P=0.0095$.

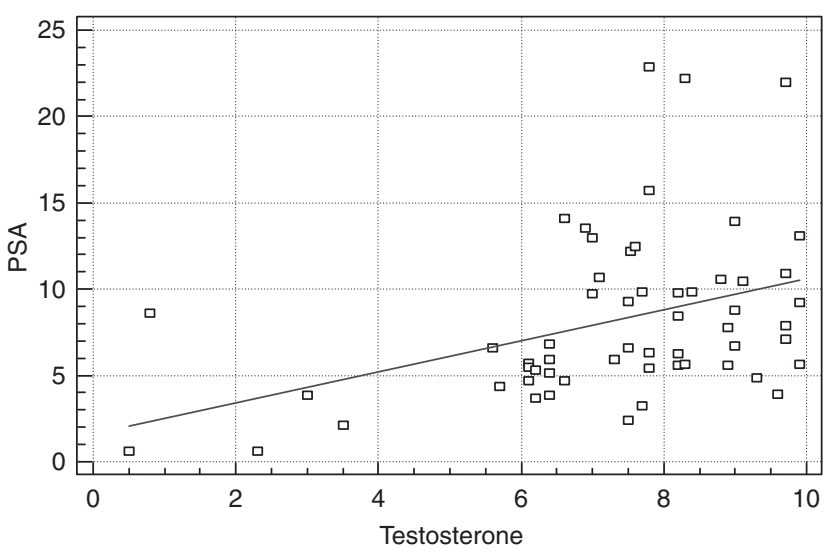

Figure 2 Correlation between testosterone and PSA in men with $T<10.4 \mathrm{nmoll}^{-1}$ ). Pearson's correlation coefficient: $r=0.3874$; $P=0.0029$.

Furthermore, UF was significantly correlated with SF $(r=0.2544 ; P<0.01$, see Figure 1$)$, such as UB with $\mathrm{SB}$ $(r=0.2512 P=0.01)$ in men with $T \geqslant 10.4 \mathrm{nmoll}^{-1}$. We did not find significant correlation between UF and SF and between UB and SB in the overall population (UF-SF: $r=0.1639 ; \quad P=0.0677 ;$ UB-SB: $r=0.1525 ; \quad P=0.0896)$, and in men with low $T$ (UF-SF: $r=-0.2412 ; P=0.3199$; UB-SB: $r=-0.3458 ; P=0.1470$ ).

Finally, serum testosterone was significantly correlated with PSA levels in men with $T<10.4 \mathrm{nmoll}^{-1}(r=0.3874$; $P=0.0029)$, whereas this correlation was missed in the whole population $(r=0.0038 ; P=0.9522)$ and in men with $T>10.4 \mathrm{nmoll}^{-1}(r=-0.0880 ; P=0.2249$, see Figure 2$)$.

\section{Discussion}

PSA levels in hypogonadal men are often low and after testosterone replacement therapy they normalize, but do not continue to increase with ongoing therapy. ${ }^{16}$

In healthy volunteers, medical castration, although dramatically reducing serum testosterone and dihydrotestosterone levels, does not abolish intraprostatic levels, and they remain at $20-30 \%$. This may indicate that there is a capacity within the prostate to manufacture and rogens in the face of very low plasma levels. ${ }^{17}$

The association between PSA and testosterone levels is not completely clarified. In this paper, we described a significant association between PSA and $T$ only in hypogonadal men $\left(T<10.4 \mathrm{nmoll}^{-1}\right)$, whereas this correlation was not evident in men with normal $T$ $\left(T>10.4 \mathrm{nmoll}^{-1}\right)$.

These data are in agreement with the 'saturation' model proposed by Morgentaler and Traish. ${ }^{1}$ According to this model, the human prostate is sensitive to massive androgen ablation but rather insensitive in normal or even in subnormal conditions (as in late-onset hypogonadism). This hypothesis has recently been confirmed in a large series of individuals consulting for ED: ${ }^{18}$ in particular it has been reported that PSA is androgendependent only in the youngest hypogonadal men, whereas this correlation disappears with higher levels of androgenization. Therefore, here we reported the same correlation in patients with PCa even if there is no evidence to support testosterone as a 'cause' of PCa. In this study, we did not analyze SHBG levels. However, in our set of analyses we could not evaluate the role of free testosterone in both urinary continence and in the setting of PCa. Further evaluation regarding this should be considered in future studies with the aim of limiting the overall cost of the trial.

Moreover, we demonstrated in a population of patients undergoing RP for PCa that men with low $T$ presented significantly worse erectile function (lower SF score) compared with men with normal $T$ : in addition, in eugonadal patients $T$ was positively correlated with sexual activity. Even though the association between $T$ and erectile function has not been completely clarified ${ }^{19-20}$ and the role of $T$ in PCa development is still under investigation, ${ }^{21,22}$ our study investigated the relationship between $T$ and ED in men with PCa, before RP. Our data seem to be in agreement with the conclusions of Khera, ${ }^{23}$ suggesting that although the relationship between testosterone and improvement in erectile function should be well established, the role of testosterone in ED recovery after RP may be of even greater significance. 
Beyond the well-known role of $T$ in regulating nitric oxide formation, recent experimental evidences have shown that $T$ also regulates the expression of phosphodiesterase type 5 (PDE5). ${ }^{24,25}$ As T positively controls both the initiation (nitric oxide synthase) and the end (PDE5) of the erectile process, its net effect on erection is modest. Hence, erections are still possible in hypogonadal conditions, in which a decreased cyclic guanosine monophosphate formation, owing to impaired nitric oxide production, is most probably counterbalanced by a reduced PDE5 activity and cyclic guanosine monophosphate hydrolysis. $^{26}$ Therefore, the main physiological action of $T$ is to timely adjust the erectile process as a function of sexual desire, finalizing erections to sex. Moreover, a trophic effect of $T$ on penile architecture has also been shown by in vivo data in different animal species and ex vivo data extrapolated from rabbits. ${ }^{24,27}$ For all the aforementioned reasons, treatment of hypogonadism restores impaired penile erections in experimental animal models, as well as in the clinical setting. Conversely, administration of testosterone to otherwise eugonadal individuals is ineffective. ${ }^{19}$

Finally, a significant result of our study is the strong correlation between urinary symptoms and SF (UF vs SF: $P=0.0095)$ in eugonadal men, and the lack of this correlation in hypogonadal men. This finding is in line with our published studies: as we previously showed in an in vitro study on the human bladder, PDE5 is expressed not only in the smooth muscle cells of the bladder wall but also in the endothelial and smooth muscle cells of the vessels. ${ }^{28}$ We also showed that the continence recovery after nerve-sparing prostatectomy for PCa was strictly correlated with recovery of SF: thus, we concluded that PDE5 inhibitors can exhibit an activity in the lower urinary tract, even in the absence of the prostatic gland, by a pathway not including prostate. ${ }^{29}$ Therefore, the androgen-dependent PDE5 activity of the bladder wall can explain the significant correlation between erectile function and urinary symptoms exclusively in patients with adequate $T$. In our experience, this activity can be provided both by the relaxation of the smooth muscle and by the improvement in perfusion of the bladder wall. ${ }^{30}$

\section{Conclusion}

We demonstrated a significant association between preoperative PSA and testosterone in men with low $T$ $\left(T<10.4 \mathrm{nmoll}^{-1}\right)$ levels before prostatectomy, and this correlation was not evident in men with normal $T$.

Moreover, we showed that before RP men with low T presented significantly worse erectile function (lower SF scores) compared with men with normal $T$, and that $T$ is positively correlated with erectile function when it is $>10.4 \mathrm{nmoll}^{-1}$.

Finally, men with normal $T$ preoperatively presented a strong correlation between their urinary continence and sexual activity.

\section{Conflict of interest}

The authors declare no conflict of interest.

\section{References}

1 Morgentaler A, Traish AM. Shifting the paradigm of testosterone and prostate cancer: the saturation model and the limits of androgen-dependent growth. Eur Urol 2009; 55: 310-320.

2 Miller NL, Bissonette EA, Bahnson R, Wilson J, Theodorescu D. Impact of a novel neoadjuvant and adjuvant hormonedeprivation approach on quality of life, voiding function, and sexual function after prostate brachytherapy. Cancer 2003; 97: 1203-1210.

3 Schroeck FR, Krupski TL, Sun L, Albala DM, Price MM, Polascik TJ et al. Satisfaction and regret after open retropubic or robot-assisted laparoscopic radical prostatectomy. Eur Urol 2008; 54: 785-793.

4 Wright J, Lin D, Cowan J, Carroll P, Litwin M, the caPSURE Investigators. Quality of life in young men after radical prostatectomy. Prost Cancer Prost Dis 2008; 11: 67-73.

5 Spry NA, Kristjanson L, Hooton B, Hayden L, Neerhut G, Gurney $\mathrm{H}$ et al. Adverse effects to quality of life arising from treatment can recover with intermittent androgen suppression in men with prostate cancer. Eur J Cancer 2006; 42: 1083-1092.

6 Calais da Silva FE, Bono AV, Whelan P, Brausi M, Marques Queimadelos A, Martin JA et al. Intermittent androgen deprivation for locally advanced and metastatic prostate cancer: results from a randomised phase 3 study of the South European Uroncological Group. Eur Urol 2009; 55: 1269-1277.

7 Tunn U. The current status of intermittent androgen deprivation (IAD) therapy for prostate cancer: putting IAD under the spotlight. BJU Int 2007; 99 (Suppl 1): 19-22.

8 Bruchovsky N, Klotz L, Crook J, Phillips N, Abersbach J, Goldenberg SL. Quality of life, morbidity, and mortality results of a prospective phase II study of intermittent androgen suppression for men with evidence of prostate-specific antigen relapse after radiation therapy for locally advanced prostate cancer. Clin Genitourin Cancer 2008; 6: 46-52.

9 Hwang TI, Lo HC, Tsai TF, Chiou HY. Association among hypogonadism, quality of life and erectile dysfunction in middle-aged and aged male in Taiwan. Int J Impot Res 2007; 19: 69-75.

10 Sarosdy MF. Testosterone replacement for hypogonadism after treatment of early prostate cancer with brachytherapy. Cancer 2007; 109: 536-541.

11 Kaufman JM, Graydon RJ. Androgen replacement after curative radical prostatectomy for prostate cancer in hypogonadal men. J Urol 2004; 172: 920-922.

12 Khera M, Lipshultz LI. The role of testosterone replacement therapy following radical prostatectomy. Urol Clin North Am 2007; 34: 549-553.

13 Rhoden EL, Averbeck MA, Teloken PE. Androgen replacement in men undergoing treatment for prostate cancer. J Sex Med 2008; 5: 2202-2208

14 Gacci M, Livi L, Paiar F, Detti B, Litwin MS, Bartoletti R et al. Quality of life after radical treatment of prostate cancer: validation of the Italian version of the University of California-Los Angeles Prostate Cancer Index. Urology 2005; 66: 338-343.

15 Rosen RC, Cappelleri JC, Smith MD, Lipsky J, Peña BM. Development and evaluation of an abridged 5 version of the International Index of Erectile Function (IIEF-5) as a diagnostic tool for erectile dysfunction. Int J Impot Res 1999; 11: 319-326.

16 Gould DC, Feneley MR, Kirby RS. Prostate-specific antigen testing in hypogonadism: implications for the safety of testosterone-replacement therapy. BJU Int 2006; 98: 1-4.

17 Page ST, Lin DW, Mostaghel EA, Hess DL, True LD, Amory JK et al. Persistent intraprostatic androgen concentrations after medical castration in healthy men. J Clin Endocrinol Metab 2006; 91: 3850-3856.

18 Corona G, Boddi V, Lotti F, Gacci M, Carini M, De Vita G et al. The relationship of testosterone to PSA in men with sexual dysfunction. J Sex Med 2010; 7: 284-292. 
19 Mikhail N. Does testosterone have a role in erectile function? Am J Med 2006; 119: 373-382.

20 Martínez-Jabaloyas JM, Queipo-Zaragozá A, Pastor-Hernández F, Gil-Salom M, Chuan-Nuez P. Testosterone levels in men with erectile dysfunction. BJU Int 2006; 97: 1278-1283.

21 Morgentaler A, Rhoden EL. Prevalence of prostate cancer among hypogonadal men with prostate-specific antigen levels of $4.0 \mathrm{ng} / \mathrm{ml}$ or less. Urology 2006; 68: 1263-1267.

22 Morote J, Ramirez C, Gómez E, Planas J, Raventós CX, de Torres IM et al. The relationship between total and free serum testosterone and the risk of prostate cancer and tumour aggressiveness. BJU Int 2009; 104: 486-489.

23 Khera M. Androgens and erectile function: a case for early androgen use in postprostatectomy hypogonadal men. J Sex Med 2009; 6 (Suppl 3): 234-238.

24 Morelli A, Corona G, Filippi S, Ambrosini S, Forti G, Vignozzi L et al. Which patients with sexual dysfunction are suitable for testosterone replacement therapy? J Endocrinol Invest 2007; 30 880-888.

25 Morelli A, Filippi S, Mancina R, Luconi M, Vignozzi L, Marini M et al. Androgens regulate phosphodiesterase type 5 expression and functional activity in corpora cavernosa. Endocrinology 2004; 145: 2253-2263.

26 Greco EA, Spera G, Aversa A. Combining testosterone and PDE5 inhibitors in erectile dysfunction: basic rationale and clinical evidences. Eur Urol 2006; 50: 940-947.

27 Traish AM, Park K, Dhir V, Kim NN, Moreland RB, Goldstein I. Effects of castration and androgen replacement on erectile function in a rabbit model. Endocrinology 1999; 140: 1861-1868.

28 Filippi S, Morelli A, Sandner P, Fibbi B, Mancina R, Marini M et al. Characterization and functional role of androgen-dependent PDE5 activity in the bladder. Endocrinology 2007; 148: 1019-1029.

29 Gacci M, Ierardi A, Delle Rose A, Tazzioli S, Scapaticci E, Filippi $S$ et al. Vardenafil can improve continence recovery after bilateral nerve sparing prostatectomy: results of a randomized, double blind, placebo controlled pilot study. J Sex Med 2010; 7: 234-243.

30 Morelli A, Filippi S, Comeglio P, Sarchielli E, Chavalmane K, Vignozzi $\mathrm{L}$ et al. Acute vardenafil administration improves bladder oxygenation in spontaneously hypertensive rats. J Sex Med 2010; 7: 59-69. 